\title{
Cutaneous Biology: In vivo blockade of pemphigus vulgaris acantholysis by inhibition of intracellular signal transduction cascades
}

I.Sánchez-Carpintero, A.España, B.Pelacho,* N.López Moratalla,* D.S.Rubenstein,† L.A.Diaz† and M.J.López-Zabalza*

Departments of Dermatology and *Biochemistry, University Clinic of Navarra, School of Medicine, University of Navarra, PO Box 4209, Pamplona, Navarra, Spain

†Department of Dermatology, University of North Carolina, Chapel Hill, NC, U.S.A.

\section{SUMMARY}

Background: Pemphigus vulgaris (PV) is an autoimmune disease characterized by mucocutaneous intraepithelial blisters and pathogenic autoantibodies against desmoglein 3. The mechanism of blister formation in pemphigus has not been defined; however, in vitro data suggest a role for activation of intracellular signalling cascades.

Objectives: To investigate the contribution of these signalling pathways to the mechanism of PV IgG-induced acantholysis in vivo.

Methods: We used the passive transfer mouse model. Mice were injected with IgG fractions of sera from a patient with PV, with or without pretreatment with inhibitors of proteins that mediate intracellular signalling cascades.

Results: Inhibitors of tyrosine kinases, phospholipase $\mathrm{C}$, calmodulin and the serinethreonine kinase protein kinase C prevented PV IgG-induced acantholysis in vivo.

Conclusions: These observations strongly support the role of intracellular signalling cascades in the molecular mechanism of PV IgG-induced acantholysis.

Key words: calmodulin, pemphigus vulgaris, phospholipase C, protein kinase C, tyrosine kinases

Correspondence: Agustin España.

E-mail: aespana@unav.es 
Pemphigus vulgaris (PV) is an autoimmune mucocutaneous disease characterized by intraepidermal blisters and epidermal-specific autoantibodies. ${ }^{1}$ These autoanti-bodies are pathogenic in passive transfer studies ${ }^{2}$ and recognize the desmosomal cadherin desmoglein (Dsg) 3. ${ }^{3}$ In approximately $50 \%$ of PV patients, a second pathogenic autoantibody recognizes Dsg1. ${ }^{4}$ Dsg1 is also the antigen bound by pemphigus foliaceus autoantibodies. ${ }^{5}$ It appears that the mucosal form of PV is characterized by a predominantly anti-Dsg3 autoimmune response, whereas the mucocutaneous form shows a combined anti-Dsg3 and anti-Dsg1 autoantibody response. ${ }^{6}$

The molecular mechanisms leading to PV IgGinduced epidermal cell detachment (acantholysis) and blister formation remain under intense investigation in several laboratories. The initiation of cell detachment by the binding of PV antibodies to the ectodomain of Dsg3 has been demonstrated in vitro; ${ }^{7}$ this process begins with binding, clustering and internalization of the PV-IgG /antigen complexes. ${ }^{8}$ The observation that PV IgG and its Fab and $\mathrm{F}(\mathrm{ab})_{2}$ fragments are also pathogenic in passive transfer studies ${ }^{9}$ led to the suggestion that antibody binding to specific epitopes of Dsg3 might sterically impair the ability of Dsg3 to function in cell-cell adhesion. ${ }^{10}$ Recent studies have mapped the pathogenic antibody binding sites to epitopes within the EC1 domain of Dsg3. ${ }^{11}$

A hypothesis supported by recent experimental data proposes that binding of PV antibodies to the ectodomain of Dsg3 triggers an intracellular signalling response in keratinocytes that leads to acantholysis. ${ }^{12,13}$ In vitro studies have demonstrated activation of the phospholipase C (PLC) pathway following the binding of PV antibodies to target antigens. ${ }^{13,14}$ PLC hydrolyses phosphatidylinositol diphosphate into diacylglycerol (DG) and inositol triphosphate (IP3). DG activates the serine/threonine kinase protein kinase C (PKC) and IP3 leads to the release of intracellular calcium. Rapid increases in IP3, intracellular calcium ${ }^{12}$ and $\mathrm{PKC}^{14,15}$ have been observed in keratinocyte cultures exposed to PV IgG. The PLC inhibitor U-73122 inhibited this response. ${ }^{13}$ Additionally, the binding of PV antibodies to keratinocytes has been associated with increased phosphorylation of Dsg3 and the dissociation of the Dsg3-plakoglobin complex, a process that may also contribute to the mechanism of acantholysis. ${ }^{16}$ Intracellular signalling induced by PV antibodies has been hypothesized to be linked to the release of plasminogen activator (PA) from keratinocytes, which in turn is proposed to lead to the degradation of cell-cell contacts; ${ }^{13,17,18}$ however, the role of PA in PV acantholysis has been challenged by studies demonstrating PV autoantibody-induced acantholysis in mice depleted of PA. ${ }^{19,20}$ Interestingly, keratinocytes derived from plakoglobin knockout mice are resistant to the pathogenic effect of PV antibodies, suggesting that the linkage of desmosomal Dsg3 to the cytoskeleton via plakoglobin might have a central role in the molecular events leading to acantholysis. ${ }^{21}$

We sought to investigate the contribution of these signalling pathways to the mechanism of acantholysis in vivo using the passive transfer mouse model. We observed that inhibitors of protein tyrosine kinases (TKs), PLC, calmodulin and the serine threonine kinase PKC prevent PV IgG-induced acantholysis in vivo. These observations provide strong support for the role of PV IgG-induced activation of signalling cascades in the molecular mechanism of acantholysis. 


\section{MATERIALS AND METHODS}

\section{Human sera}

Serum samples were obtained from a patient with mucocutaneous PV, during states of both active disease (PV1a) and disease remission (PV1b), and from a normal human donor. Patient and control sera were tested by indirect immunofluorescence (IF) using monkey oesophagus as a tissue substrate. The immunological characteristics of these sera are presented in Table 1.

\section{IgG purification}

IgG fractions of sera from the patient with PV and the normal human donor were prepared as previously described. ${ }^{2,22}$ Briefly, IgG fractions were precipitated with $50 \%$ ammonium sulphate followed by affinity chromatography on Staphylococcus protein A conju-gated to agarose beads. Bound IgG was eluted with $0.2 \mathrm{~mol} \mathrm{~L}^{-1}$ glycine/ $\mathrm{HCl}, \mathrm{pH} 3$, dialysed extensively against phosphate-buffered saline (PBS), $\mathrm{pH} 7 \cdot 4$, concentrated by ultrafiltration (Millipore/Amicon, Beverly, MA, U.S.A.), filter-sterilized, and stored at $70{ }^{\circ} \mathrm{C}$ until use. Protein concentrations were determined by Bradford assay. ${ }^{23}$ The IgG concentration in each fraction was estimated by nephelometry using goat antihuman IgG (Beckman Array 360 System, Holliston, MA, USA).

\section{Detection of antidesmoglein 3 antibodies by immunoprecipitation}

The anti-Dsg3 activity of the PV patient sera was assayed by immunoprecipitation (IP) of recombinant Dsg3 (rDsg3). ${ }^{4}$ Three microlitres of each serum were incubated with $3 \mu \mathrm{g}$ of rDsg3 for $1 \mathrm{~h}$ at room temperature followed by addition of protein A-conjugated agarose beads, and subjected to extensive washing with $0.1 \%$ Triton X-100 in Tris-buffered saline containing $3 \mathrm{mmol} \mathrm{L}^{-1}$ of calcium prior to elution of the immunoprecipitates from the beads with Laemmli sample buffer [ $2 \%$ sodium dodecyl sulphate (SDS), $0.1 \mathrm{~mol} \mathrm{~L}^{-1}$ Tris, $\mathrm{pH}$ 6.8]. Immunoprecipitates were separated by $10 \%$ SDS-polyacrylamide gel electrophoresis, transferred to nitrocellulose membrane, and probed by Western blot analysis using rabbit antihuman Dsg3 primary antibodies (1:2000) and horseradish peroxidase-conjugated goat antirabbit IgG secondary antibodies (1:3000). The immunoreactants were developed using the enhanced chemiluminescence reaction (ECLPlus system; Amersham Pharmacia Biotech, St Albans, U.K.).

\section{Detection of antidesmoglein 3 antibodies by enzyme-linked immunosorbent assay}

PV sera were tested by two enzyme-linked immunosorbent assay (ELISA) techniques. In the first, recombinant histidine-tagged Dsg3 protein encompassing the ectodomain of Dsg3 was expressed in baculovirus ${ }^{4}$ and afinity-purfied by Ni-agarose chromatography. Microtitre Ni-coated plates were incubated with $1 \mu \mathrm{g}$ per well of rDsg3, blocked with $0.2 \%$ bovine serum albumin, washed extensively with $0.5 \%$ Tween 20 in Tris buffer, $\mathrm{pH}$ 7.3, and used to test the patient and control sera for the presence of antiDsg3 reactivity in ELISA. $^{24}$ The second protocol utilized a commercially available ELISA kit to test for 
anti-Dsg3 antibodies according to the manufacturer's recommendations (RhiGene Inc., Des Plaines, IL, U.S.A.). The ELISA results are given in arbitrary units as follows: negative ( $£ 9$ units), indeterminate (10-20 units) and positive for anti-Dsg3 (> 20 units). The results obtained from both ELISA techniques were comparable; data are shown for the more readily available commercial kit.

\section{Testing of IgG pathogenicity using the passive transfer animal model}

Each IgG fraction was tested for pathogenicity by passive transfer experiments as previously described. ${ }^{2,22}$ IgG from PV1a and PV1b sera was injected intradermally (4 mg $\mathrm{g}^{-1}$ body weight) into neonatal Balb/C mice ( $\mathrm{n}=3$ for each $\operatorname{IgG}$ ) and evaluated $24 \mathrm{~h}$ later for evidence of skin blisters. Following examination of the skin for blisters, animals were killed, and serum was collected for indirect IF assays, using human skin as substrate. Skin biopsies from each animal included lesional skin samples for routine histological examination by haematoxylin and eosin staining and perilesional skin for direct IF studies.

\section{Investigation of the role of inhibitors of signalling pathways on pemphigus vulgaris IgG-induced skin disease in mice}

Inhibitors of proteins that mediate intracellular signalling cascades were tested for their ability to prevent PV IgG-induced blistering in the passive transfer mouse model. Nontoxic doses of the inhibitors genistein (Sigma, St Louis, MO, U.S.A.), herbimycin (Sigma), U-73122 (Biomol, Plymouth Meeting, PA, U.S.A.), bisindolylmaleinamide (Boehringer Mannheim, Mannheim, Germany) and W-7 hydrochloride (Calbiochem, La

Jolla, CA, U.S.A.) were selected based on in vitro studies reported elsewhere. ${ }^{14,18}$ Additionally, lethality studies carried out in our laboratory demonstrated no increased mortality in the mice at $48 \mathrm{~h}$ postinjection at inhibitor doses used in this study (data not shown). Inhibitors were administered by intradermal injection $3 \mathrm{~h}$ before intradermal injection of PV IgG. Inhibitors were diluted in PBS and injected into mice ( $\mathrm{n}=3$ for each inhibitor) at the following doses: the TK inhibitor genistein, $500 \mu \mathrm{g} \mathrm{g}^{-1}$ body weight; the TK inhibitor herbimycin, $15 \mu \mathrm{g} \mathrm{g}^{-1}$ body weight; the PLC inhibitor U-73122, $100 \mu \mathrm{g} \mathrm{g}^{-1}$ body weight; the PKC inhibitor bisindolylmaleinamide, $100 \mathrm{\mu g} \mathrm{g}^{-1}$ body weight; and the calmodulin antagonist W-7 hydrochloride, $200 \mu \mathrm{g} \mathrm{g}^{-1}$ body weight.

\section{RESULTS}

PV1a IgG recognizes desmoglein 3 and induces acantholysis in vivo by passive transfer to neonatal mice

The IgG fraction from the patient with PV during the active phase of the disease (PV1a) was immunologically characterized for its ability to target Dsg3 specifically. Indirect IF, IP and ELISA assays using rDsg3 were used to assay for the presence and titre of Dsg3- 
speciic IgG in each serum sample. Sera from the PV patient, but not from the normal human control, recognized rDsg3 (Table 1, Fig. 1).

Passive transfer experiments in neonatal mice were used to assay for the ability of each IgG sample to induce acantholysis in vivo. IgG from normal human serum failed to induce acantholysis by passive transfer studies in neonatal mice using two different doses of IgG (4 and $8 \mathrm{mg} \mathrm{g}^{-1}$ body weight) (Table 2). In contrast, IgG from PV1a (active disease) was pathogenic at $4 \mathrm{mg} \mathrm{g}^{-1}$ body weight; IgG collected from the same patient during disease remission (PV1b) was not pathogenic (Table 2, Fig. 2).

\section{Pretreatment of mice with inhibitors of intracellular signalling pathways blocks the pathogenic effect of PV1a IgG}

As shown in Table 2 and Figure 2, the pathogenic PV1a IgG failed to induce acantholysis in mice pretreated with the TK inhibitor genistein, the TK inhibitor herbimycin, the PLC inhibitor U-73122, the PKC inhibitor bisindolylmaleinamide or the calmodulin antagonist W-7 hydrochloride. Control mice pretreated with PBS followed by PV1a developed extensive disease similar to animals injected with only PV1a IgG.

\section{DISCUSSION}

Pretreatment of mice with inhibitors of intracellular signalling cascades prevented PV IgG-induced blister formation in vivo. PV1a IgG was selected to conduct these studies because it induced obvious and reproducible disease in neonatal mice and sufficient quantities of this serum could be obtained to perform the necessary experiments and controls in triplicate. In addition to anti-Dsg3 antibodies, approximately 50\% of PV patient sera contain a second pathogenic autoantibody that recognizes Dsg1. ${ }^{4}$ Although we have characterized the Dsg3 autoantibodies in the PV1a serum, we have not excluded the presence in this serum of Dsg1 autoanti-bodies. On the basis of the generated data, we cannot formally determine whether the observed inhibition of blister formation occurred via inhibition of anti-Dsg1 andor anti-Dsg3 antibody-mediated acantholysis; however, both Dsg1- and Dsg3-specific autoantibodies affinity-purified from human sera are each, by them-selves, capable of inducing acantholysis in the passive transfer mouse model. ${ }^{6}$ As such, it is not necessary to invoke the presence of Dsg1-specific autoantibodies in the mucocutaneous PV1a serum as being necessary for acantholysis in the passive transfer mouse model.

Blisters did not develop when mice were pretreated with the TK inhibitors genistein and herbimycin, the PLC inhibitor U-73122, the calmodulin inhibitor $\mathrm{W}-7$, or the PKC inhibitor bisindolylmaleinamide. Inhibitorand noninhibitor-treated mice demonstrated comparable serum levels of IgG by indirect IF, indicating that pretreatment with inhibitors did not impair the entry of IgG from the site of injection into the systemic circulation. Additionally, the inhibitors did not affect the ability of pathogenic PV IgG to bind to murine epidermis. Direct IF of skin biopsies from mice pretreated with inhibitors prior to injection of pathogenic IgG was similar to that of mice that had not been 
pretreated; both groups demonstrated IgG staining at the keratinocyte cell membrane. In contrast, inhibitorpretreated mice did not demonstrate acantholysis, indicating that the effect of the inhibitors in inhibiting blister formation was downstream of the binding of pathogenic IgG to the keratinocyte cell surface.

The molecular mechanisms of acantholysis have yet to be defined; however, an increasing body of data suggests that propagation of signals initiated across the cell membrane may have a role in PV IgG-mediated acantholysis. ${ }^{25}$ Our finding that several inhibitors of intracellular signalling cascades inhibit PV IgG acan-tholysis, whether or not the PV serum is composed of only anti-Dsg3 autoantibodies or both anti-Dsg3 and antiDsg1 autoantibodies, is consistent with a mechanism in which signalling events contribute to the loss of cell-cell adhesion. These inhibitors may, in fact, define specific steps in the propagation of PV IgGinitiated signalling. Ligandreceptor interactions trigger TK activity leading to PLC activation, which in turn activates the phosphatidylinositol4,5-diphosphate pathway ${ }^{26}$ leading to generation of IP3 and DG. Subsequent increases in intracellular calcium activate calmodulin and then serine threonine kinases such as PKC and the CAM kinases. The TK inhibitors genistein and herbimycin may inhibit acantholysis by disrupting upstream elements of this signalling cascade. Similarly, it is not surprising that inhibition of downstream elements, including PLC, calmodulin and PKC by U-73122, W-7 and bisindolylmaleinamide, respectively, also inhibits acantholysis. Taken together, these data support the role of this signalling cascade in the development of PV IgG-induced acantholysis.

The present studies show that in vivo administration of U-73122 prevents the development of acantholysis, confirming the in vitro findings made by others. ${ }^{13}$ Although different isoforms of PLC are inhibited by U-73122, inhibition by genistein and herbimycin suggests a role for PLCy in mediating PV IgG signalling. The PKC inhibitor bisindolylmaleinamide has been shown to alter the assembly/disassembly of the desmosomes, ${ }^{26-28}$ possibly due to changes in phosphorylation of proteins such as desmoplakin, ${ }^{29,30}$ plakoglobin or Dsg3. ${ }^{16}$

In conclusion, the data demonstrate a role for intracellular signal transduction cascades in the devel-opment of PV IgG-induced loss of cell-cell adhesion. In vivo inhibition of acantholysis in the mouse model of PV by inhibition of intracellular signalling demonstrates the value of targeting these pathways for the treatment of PV in humans.

\section{ACKNOWLEDGMENTS}

L.A.D. and D.S.R. are supported by USPHS grants RO1 AR32599, RO1 AR32081 (L.A.D.) and RO1 AI49427 (D.S.R.) from the NIH. This work was supported by 'Fundación Echébano', Spain. B.P. was the recipient of a fellowship from the Government of Navarra, Spain. 


\section{REFERENCES}

1. Stanley JR. Pemphigus and pemphigoid as paradigms of organ-specific, autoantibody-mediated diseases. J Clin Invest 1989; 83: 1443-8.

2. Anhalt GJ, Labib RS, Voorhees JJ et al. Induction of pemphigus in neonatal mice by passive transfer of IgG from patients with the disease. N Engl J Med 1982; 306: 1189-96.

3. Amagai M, Klaus-Kovtun V, Stanley JR. Autoantibodies against a novel epithelial cadherin in pemphigus vulgaris, a disease of cell adhesion. Cell 1991; 67: 869-77.

4. Ding X, Aoki V, Mascaro JM Jr et al. Mucosal and mucocutaneous (generalized) pemphigus vulgaris show distinct autoantibody profiles. J Invest Dermatol 1997; 109: 592-6.

5. Eyre RW, Stanley JR. Human autoantibodies against a desmo-somal protein complex with a calcium-sensitive epitope are characteristic of pemphigus foliaceus patients. J Exp Med 1987; 160: 1719-24.

6. Ding X, Diaz LA, Fairley JA et al. The anti-desmoglein 1 auto-antibodies in pemphigus vulgaris sera are pathogenic. J Invest Dermatol 1999; 112: 739-43.

7. Karpati SM, Amagai $R$, Prussick $K$ et al. Pemphigus vulgaris antigen, a desmoglein type cadherin, is localized within kera-tinocyte desmosome. J Cell Biol 1993; 122: 409-15.

8. Patel HP, Diaz LA, Anhalt GJ et al. Demonstration of pemphigus antibodies on the cell surface of murine epidermal cell monolayers and their internalization. $\mathrm{J}$ Invest Dermatol 1984; 83: 409-15.

9. Mascaro JM Jr, España A, Liu Z et al. Mechanisms of acantholysis in pemphigus vulgaris: role of IgG valence. Clin Immunol Immunopathol 1997; 85: 90-6.

10. Diaz LA, Marcelo CL. Pemphigus and pemphigoid antigens in cultured epidermal cells. Br J Dermatol 1978; 98: 631-7.

11. Sekiguchi M, Futei Y, Fujii Y et al. Dominant autoimmune epitopes recognized by pemphigus antibodies map to the $\mathrm{N}$-ter-minal adhesive region of desmogleins. J Immunol 2001; 167: 5439-48.

12. Seishima M, Esaki C, Osada $\mathrm{K}$ et al. Pemphigus IgG, but not bullous pemphigoid IgG, causes a transient increase in intracel-lular calcium and inositol 1,4,5-triphosphate in DJM-1 cells, a squamous cell carcinoma line. J Invest Dermatol 1995; 104: 33-7.

13. Esaki C, Seishima M, Yamada T et al. Pharmacologic evidence for involvement of phospholipase C in pemphigus IgG-induced inositol 1,4,5-triphosphate generation, intracellular calcium increase, and plasminogen activator secretion in DJM-1 cells, a squamous cell carcinoma line. J Invest Dermatol 1995; 105: 32933.

14. Seishima M, Iwasaki-Bessho $\mathrm{Y}$, Itoh $\mathrm{Y}$ et al. Phosphatidylcholinespecific phospholipase $\mathrm{C}$, but not phospholipase $\mathrm{D}$, is involved in pemphigus IgGinduced signal transduction. Arch Dermatol Res 1999; 291: 606-13. 
15. Osada K, Seishima M, Kitajima Y. Pemphigus IgG activates and translocates protein kinase $\mathrm{C}$ from the cytosol to the particulate / cytoskeleton fractions in human keratinocytes. J Invest Dermatol 1997; 108: 482-7.

16. Aoyama Y, Owada MK, Kitajima Y. A pathogenic autoantibody, pemphigus vulgaris-IgG, induces phosphorylation of desmoglein 3, and its dissociation from plakoglobin in cultured keratinocytes. Eur J Immunol 1999; 29: 2233-40.

17. Schaefer BM, Jaeger C, Kramer MD. Plasminogen activator sys-tem in pemphigus vulgaris. Br J Dermatol 1996; 135: 726-32.

18. Seishima M, Satoh S, Nojiri $M$ et al. Pemphigus IgG induces expression of urokinase plasminogen activator receptor on the cell surface of cultured keratinocytes. J Invest Dermatol 1997; 109: 650-5.

19. Anhalt GJ, Patel HP, Labib RS et al. Dexamethasone inhibits plasminogen activator activity in experimental pemphigus in vivo but does not block acantholysis. J Immunol 1986; 136: 113-17.

20. Mahoney MG, Wang ZH, Stanley JR. Pemphigus vulgaris and pemphigus foliaceus antibodies are pathogenic in plaminogen activator knockout mice. $\mathrm{J}$ Invest Dermatol 1999; 113: 22-5.

21. Caldelari R, de Bruin A, Baumann D et al. A central role for the armadillo protein plakoglobin in the autoimmune disease pem-phigus vulgaris. $\mathrm{J}$ Cell Biol 2001; 153: 823-34.

22. España A, Diaz LA, Mascaro JM Jr et al. Mechanisms of acan-tholysis in pemphigus foliaceus. Clin Immunol Immunopathol 1997; 85: 83-9.

23. Bradford MM. A rapid and sensitive method for quantitation of microgram quantities of protein utilizing the principle of proteindye binding. Anal Biochem 1976; 72: 248-54.

24. Warren SJP, Lin MS, Giudice GJ et al. The prevalence of antibodies against desmoglein 1 in endemic pemphigus foliaceus in Brazil. N Engl J Med 2000; 343: 23-30.

25. Kitajima Y, Aoyama Y, Seishima M. Transmembrane signaling for adhesive regulation of desmosomes and hemidesmosomes, and for cell-cell detachment induced by pemphigus IgG in cultured keratinocytes: involvement of protein kinase C. J Invest Dermatol Symp Proc 1999; 4: 137-44.

26. Citi S. Protein kinase inhibitors prevent junction dissociation induced by low extracellular calcium in MDCK epithelial cells. J Cell Biol 1992; 117: 169-78.

27. Kitajima Y, Aoyama Y, Osada K et al. Substrates for phosphorylation by protein kinase $\mathrm{C}$ activated with pemphigus-IgG in cultured keratinocytes. J Invest Dermatol 1996; 106: 847 (Abstr.).

28. Amar LS, Shabana AH, Oboeuf $\mathrm{M}$ et al. Desmosomes are regulated by protein kinase C in primary rat epithelial cells. Cell Adhes Commun 1998; 5: 1-12.

29. Amar LS, Shabana AH, Oboeuf $\mathrm{M}$ et al. Involvement of des-moplakin phosphorylation in the regulation of desmosomes by protein kinase $\mathrm{C}$, in HeLa cells. Cell Adhes Commun 1999; 7: 125-38.

30. Kowalewski C, Kaiser HW, Majewski S et al. Protein kinase H7 prevents the acantholysis induced by pemphigus antibodies. Eur J Dermatol 1994; 4: 238-42. 
Table 1. Characteristics of IgG fractions

\begin{tabular}{|l|c|c|c|}
\hline Sera & Indirect IF titre (MO) & IP (rDsg3) & ELISA (rDsg3) \\
\hline PV1a & $1: 80$ & Positive & Not done \\
\hline PV1b & Negative & Not done & 39 units \\
\hline Normal human serum & Negative & Negative & Negative \\
\hline
\end{tabular}

IF, immunofluorescence; MO, monkey oesophagus; IP, immunoprecipitation;

rDsg3, recombinant desmoglein 3; ELISA, enzyme-linked immunosorbent assay;

$\mathrm{PV}$, pemphigus vulgaris.

Table 2. Testing pathogenic PV1 IgG by passive transfer in mice

\begin{tabular}{|l|c|c|c|c|c|}
\hline Mice injected with: & $\begin{array}{c}\text { No. of } \\
\text { mice } \\
\text { tested }\end{array}$ & $\begin{array}{c}\text { Skin } \\
\text { blister } \\
\text { s }\end{array}$ & $\begin{array}{c}\text { Histology } \\
\text { (haematoxylin } \\
\text { and eosin) }\end{array}$ & Direct IF & $\begin{array}{c}\text { Indirect } \\
\text { IF }\end{array}$ \\
\hline Normal IgG & 3 & Absent & Normal & Negative & Negative \\
\hline PV1a IgG & 3 & +++ & Acantholysis & +++ & $1: 40$ \\
\hline PV1b IgG & 3 & Absent & Normal & Negative & Negative \\
\hline PV1a IgG + PBS & 3 & +++ & Acantholysis & +++ & $1: 80$ \\
\hline PV1a IgG + genistein & 3 & Absent & Normal & +++ & $1: 40$ \\
\hline PV1a IgG + herbimycin & 3 & Absent & Normal & +++ & $1: 20$ to \\
& 3 & Absent & Normal & +++ & $1: 40$ to \\
\hline $\begin{array}{l}\text { PV1a IgG + } \\
\text { bisindolylmaleinamide }\end{array}$ & 3 & Absent & Normal & +++ & $1: 80$ \\
\hline PV1a IgG + U-73122 & 3 & Absent & Normal & +++ & $\begin{array}{c}1: 20 \text { to } \\
1: 40\end{array}$ \\
\hline PV1a IgG + W-7 & & & & & \\
\hline
\end{tabular}

IF, immunofluorescence; PV, pemphigus vulgaris; PBS, phosphate-buffered saline. 


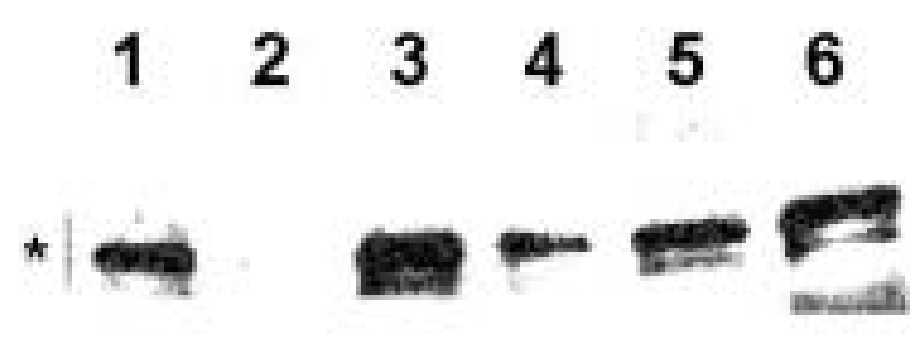

Figure 1. Detection of antidesmoglein (antiDsg) 3 autoantibodies by immunoprecipitation of recombinant Dsg3 (rDsg3). rDsg3 is shown in lane 1. A negative control serum (lane 2), a positive control pemphigus vulgaris (PV) serum (lane 3) and the PV1 test serum (lane 4) were incubated with rDsg3 and precipitated with protein A-agarose beads. The immunoprecipitates were fractionated by $10 \%$ sodium dodecyl sulphate-polyacrylamide gel electrophoresis and blotted to nitrocellulose. To detect precipitated rDsg3, the blots were probed with rabbit anti-Dsg3 primary antibodies, goat antirabbit IgG horseradish peroxidase-conjugated secondary antibodies, and developed by enhanced chemiluminescence. The 66-kDa ectodomain of rDsg3 is indicated by an asterisk. All PV sera recognized rDgs3. 
Clinical findings

PV1a
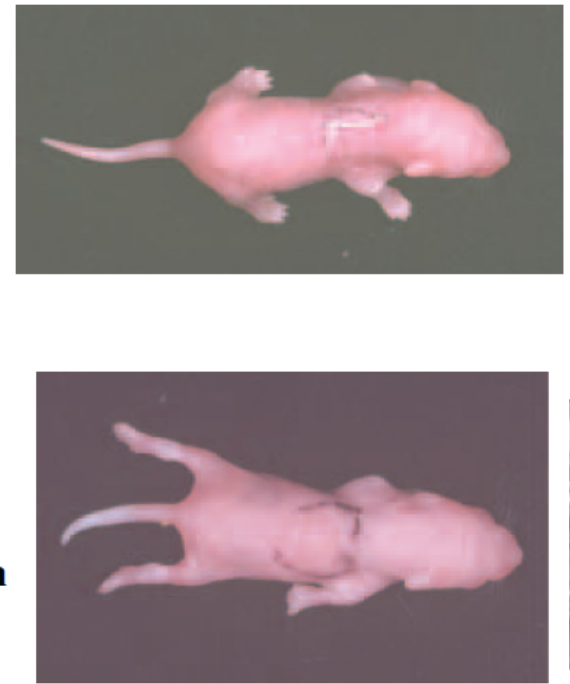

Histopathology

Direct

immunofluorescence
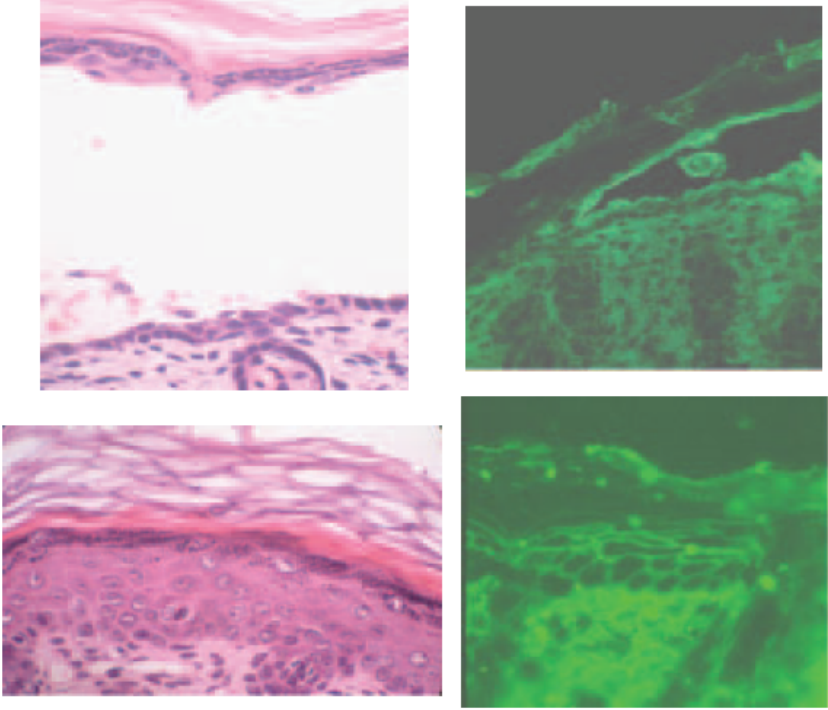

Figure 2. Inhibition of intracellular signalling cascades prevents pemphigus vulgaris (PV) IgG blister formation in vivo. The clinical, histological and immunofluorescent characteristics of neonatal mice injected with PV IgG (PV1a) were compared with those in mice pretreated with the tyrosine kinase inhibitor herbimycin prior to injection of pathogenic PV IgG (PV1a + herbimycin). Gross blister formation and histological acantholysis are seen in the PV-treated mice. Pretreatment with herbimycin prevents both gross and histological evidence of blister formation (black ink marks the PV serum injection site). Direct immunofluorescence staining of punch biopsies demonstrates cell surface staining for PV IgG in both PV IgG- and PV IgG + herbimycin-treated mice, demonstrating that pretreatment with herbimycin did not affect the in vivo desmoglein 3 binding of the pathogenic IgG. Similar results to that observed in the herbimycinpretreated mice were obtained in mice pretreated with genistein, U-73122, bisindolylmaleinamide and W-7 hydrochloride. 